\section{A role for calreticulin in the adult heart?}

\author{
Alexander Maass and Leslie A. Leinwand \\ Department of Molecular, Cellular, and Developmental Biology, University of Colorado, \\ Boulder, Colorado, USA \\ Address correspondence to: Leslie A. Leinwand, Department of Molecular, Cellular, and Developmental Biology, University of Colorado, \\ Campus Box 347, Boulder, Colorado 80309-0347, USA. Phone: (303) 492-7606; Fax: (303) 492-8907; E-mail: leinwand@stripe.colorado.edu.
}

The essential role of calcium in cardiac development, function, and disease is incontrovertible. The multiple pathways that regulate $\mathrm{Ca}^{2+}$ homeostasis in the heart are complex and frequently intersecting, involving a large number of myocyte proteins. One protein that is emerging as having a central role in $\mathrm{Ca}^{2+}$ homeostasis and in cardiac development is the ubiquitous chaperone protein of the endoplasmic reticulum (ER), calreticulin. Over 40 different functions have been ascribed to this $\mathrm{Ca}^{2+}$-binding protein, including regulation of gene expression, protein folding, cell adhesion, and autoimmunity (reviewed in ref. 1). In the mouse heart, calreticulin expression is activated at 9.5 days postcoitum (dpc) and remains high until $14.5 \mathrm{dpc}$. Expression is relatively low by $18 \mathrm{dpc}$ and is barely detectable postnatally. Mice in which the Calreticulin gene has been inactivated die by $14.5 \mathrm{dpc}$, primarily due to cardiac defects with a marked decrease in ventricular wall thickness (2). Therefore, the importance of calreticulin in cardiac development has been solidified, although its specific

In this issue of $J C I$, Nakamura and colleagues (3) have forced expression of calreticulin in adult cardiac myocytes in the mouse, to study the sequelae of sustained high expression of this protein after development. These animals exhibit decreased systolic function, chamber dilation, and wall thinning, and they die of sudden cardiac death (3). This premature death is associated with complete heart block. While the mechanisms behind these phenotypes remain somewhat unclear, there is a significant decrease in inward $\mathrm{Ca}^{2+}$ current as well as a decrease in expression of connexins 40 and 43 . Consistent with a role for the connexins in the phenotypes of the mice is the finding that inactivation of connexin 43 in adult cardiac role remains unclear. myocytes results in severe arrhythmias (4). In addition, mice lacking connexin40 exhibit sinoatrial, intraatrial, and atrioventricular conduction disturbances (5). The complete heart block in calreticulin-overexpressing mice might therefore be explained, at least in part, by secondary changes in expression of other proteins, including the connexins.

Since other proteins that have been implicated in cardiac pathogenesis, including SERCA2, phospholamban, calsequestrin, and $\beta$-myosin heavy chain, remain unchanged in this model, the decrease in connexins 40 and 43 appears to be a relatively specific consequence of expressing the transgene. Still, reduced connexin levels seem unlikely to explain all of its effects, since the conditional and complete null mice for connexins 40 and 43 , respectively, do not exhibit dilation and systolic dysfunction.

\section{Commentary}

See related article, pages $1245-1253$
These interesting findings raise issues about the potential role for calreticulin in the pathogenesis of human arrhythmias.

Several recent reviews $(1,6)$ have sifted through the multitude of roles ascribed to calreticulin, seeking to clarify the relevant functions of this protein. Calreticulin binds $\mathrm{Ca}^{2+}$ through two functionally distinct sites and is localized to the ER, where it is known to function as a lectinlike chaperone for a large number of proteins. One might speculate that calreticulin is involved in the proper folding of these proteins, as has been demonstrated for other membrane-associated proteins (6). However, the mechanism by which calreticulin ensures proper protein folding and the role that $\mathrm{Ca}^{2+}$ plays in this process remain unknown. Given that the ER is one of the largest organelles of the cell and that it is

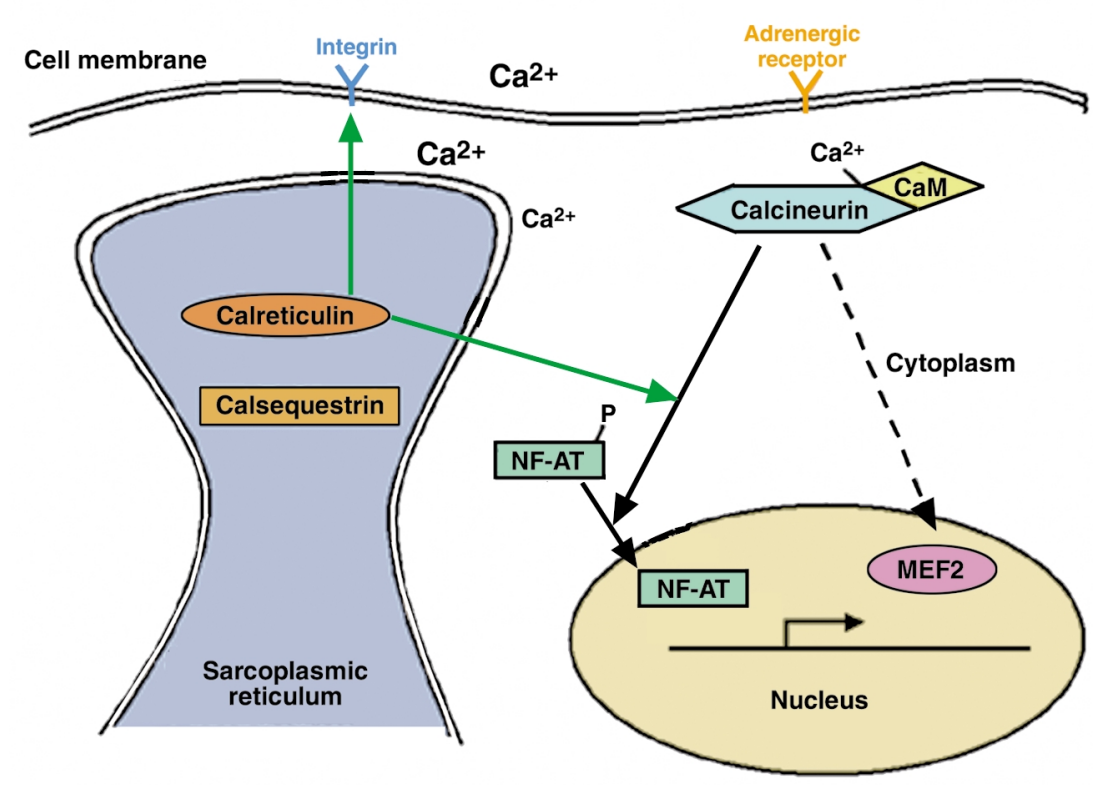

Figure 1

Involvement of calreticulin in signal transduction pathways. Calreticulin is essential for integrin signaling and the dephosphorylation and nuclear translocation of NF-AT3. The size of the $\mathrm{Ca}^{2+} \mathrm{sym}-$ bols reflects the differing concentrations in the extracellular space and the sarcoplasmic reticulum $\left(10^{-3} \mathrm{M}\right)$, as compared with the cytoplasm $\left(10^{-} 5 \mathrm{M}\right.$ in systole and $10^{-7} \mathrm{M}$ in diastole, with higher levels seen in $\mathrm{Ca}^{2+}$ sparks). 
arguably the most important source of intracellular $\mathrm{Ca}^{2+}$, calreticulin is well situated to play an important role in cardiac cell biology.

\section{Calreticulin deficiency and overexpression}

Mice with targeted disruption of the Calreticulin gene die in utero with decreased ventricular cell mass due to increased apoptosis of cardiac myocytes (7). Calreticulin-deficient cells show reduced nuclear import of the transcription factor nuclear factor of activated transcription 3 (NF-AT3), indicating that calreticulin participates in the $\mathrm{Ca}^{2+} /$ calcineurin/NFAT/GATA4 signal transduction pathway (2). Integrin-mediated $\mathrm{Ca}^{2+}$ signaling and cell adhesion are also impaired in calreticulin-deficient cells (8) (Figure 1). Mice with targeted disruption of GATA4 or NFATc also show impairment of early cardiac development, but their phenotypes are distinct from those of the Calreticulin null mice. Mice null for GATA4 fail heart tube formation and die between 8.5 and $10.5 \mathrm{dpc}$ (9). Mice lacking NFATc fail to form normal cardiac valves and die from circulatory failure around $14.5 \mathrm{dpc}(10,11)$. Therefore, the embryonic lethality of the calreticulin-deficient mice cannot be explained simply by the disruption of NF-AT/GATA4 pathways.

The other main $\mathrm{Ca}^{2+}$-binding chaperone of the sarcoplasmic reticulum $(\mathrm{SR})$ is calsequestrin, a protein that is expressed in higher amounts in the adult heart than calreticulin. Overexpression of calsequestrin in the mouse heart is associated with yet another phenotype, involving cardiac hypertrophy and decreased contractility. Hence, the two chaperones likely carry out at least some distinct functions within the SR (12).

\section{Calreticulin and human heart disease}

Several studies have shed light on gene expression changes in several forms of heart disease, including heart failure, familial hypertrophic cardiomyopathy, and primary dilated cardiomyopathy (reviewed in ref. 13). Changes in the expression of calciumtransporting proteins (such as SERCA and the $\mathrm{Na}^{+} / \mathrm{Ca}^{2+}$ exchanger) and their regulators (such as phospholamban) have been observed in many forms of acquired and genetic heart diseases, most notably in cardiac hypertrophy and heart failure (reviewed in ref. 14). These changes seem to be secondary to the primary cardiac dysfunction, but recent publications have implicated these changes as major contributors to systolic and diastolic dysfunction. In fact, targeted disruption of the Phospholamban gene seems to rescue or delay pathogenesis in several mouse models of dilated and hypertrophic cardiomyopathy $(15,16)$. In addition to the obvious role of $\mathrm{Ca}^{2+}$ in the cardiac contraction cycle, several calciumdependent enzymes such as the phosphatase calcineurin and the family of $\mathrm{Ca}^{2+} /$ calmodulin-dependent kinases have been implicated in the development of cardiac hypertrophy and heart failure (reviewed in ref. 17). $\mathrm{Ca}^{2+}$ levels change rapidly in the cytoplasm during contraction and relaxation, but there are also localized, subcellular changes known as $\mathrm{Ca}^{2+}$ sparks (18). Ion channels as well as calciumbinding proteins such as calsequestrin and calreticulin are important in the regulation of global cytosolic as well as localized $\mathrm{Ca}^{2+}$ concentrations in the different cell compartments (Figure 1). This suggests a role for $\mathrm{Ca}^{2+}$-regulating proteins in multiple cellular processes, such as signal transduction and cell growth. To date, there are no reports quantifying calreticulin levels in human heart disease.

Calreticulin has been implicated in congenital heart block in humans, since autoantibodies against calreticulin have been identified in a subset of patients (19). Because the exact physiological function of this protein in the adult heart remains obscure, the pathophysiological relevance of autoantibodies is also unclear. The transgenic mice described in the present work overexpress calreticulin and have a dilated cardiomyopathy, but they also exhibit a complete heart block, further obscuring calreticulin's roles in these disease pathways. However, congenital heart block and cardiomyopathy might share common molecular mechanisms, as suggested by recent evidence that patients with congenital heart block are prone to dilated cardiomyopathy even when their cardiac conduction is restored by an artificial pacemaker (20).

Studies with calreticulin-deficient cells suggest that this protein participates in signal transduction pathways involving integrins or calcineurin, which are thought to drive myocyte hypertrophy and other pathophysiological changes in the heart. The normal adult myocardium, however, expresses only low levels of calreticulin, raising the possibility that calreticulin is induced with the fetal gene program that is reactivated during cardiac hypertrophy and failure. However, other fetal gene products normally

Table 1

Phenotypes of transgenic mice with alterations in connexin 40 , connexin 43 , calsequestrin, or calreticulin

\begin{tabular}{|c|c|c|c|c|c|c|}
\hline Gene product & $\begin{array}{l}\text { Overexpression }(\mathrm{O}) \\
\text { Knockout }(\mathrm{K})\end{array}$ & $\begin{array}{l}\text { Conduction } \\
\text { velocity }\end{array}$ & $\begin{array}{c}\text { Sudden } \\
\text { cardiac death }\end{array}$ & $\begin{array}{c}\text { Effects on } \\
\text { gene expression }\end{array}$ & Arrhythmia & Contractility \\
\hline Calreticulin & $\mathrm{O}$ (heart) & $\downarrow \downarrow \downarrow$ & + & $\begin{array}{c}\text { Cx43, Cx40 } \downarrow \downarrow \\
\text { ANF, BNP } \uparrow\end{array}$ & $\begin{array}{c}\text { Complete heart } \\
\text { block }\end{array}$ & $\downarrow$ \\
\hline Calsequestrin & $\mathrm{O}$ (heart) & ND & - & $\begin{array}{c}\text { Calreticulin } \uparrow \\
\text { SERCA, PLB } \uparrow \\
\text { ANF, } \beta-\mathrm{MHC} \uparrow\end{array}$ & ND & $\downarrow \downarrow$ \\
\hline Connexin 40 & $\mathrm{~K}$ & $\downarrow \downarrow \downarrow$ & - & $\mathrm{Cx} 43 \leftrightarrow$ & Atrial & ND \\
\hline Connexin 43 & $\mathrm{~K}$ (conditional, heart) & $\downarrow \downarrow \downarrow$ & + & ND & Ventricular & $\leftrightarrow$ \\
\hline
\end{tabular}

Cx, connexin; ANF, atrial natriuretic factor; BNP, brain natriuretic peptide; PLB, phospholamban; $\beta$-MHC, $\beta$-myosin heavy chain; ND, not determined. 
induced in hypertrophy are not found in the overexpressing mice. To more accurately study the consequences of calreticulin re-expression in the adult heart, an inducible expression system might be developed to turn expression on and off at later time points.

The intriguing overlap in the phenotypes of calreticulin- or calsequestrinoverexpressing mice with mice carrying targeted disruption of one of the Connexin genes (summarized in Table 1) makes a compelling case for examining the expression of these genes in human heart disease. Postnatal development of cardiac conduction is remarkably different between mice and humans, in that heart rate increases with age in mice, whereas it decreases with age in humans. The molecular mechanisms underlying acquired conduction disorders (other than for ischemia-related conduction blocks) remain completely obscure. Myocardial biopsies taken from patients undergoing pacemaker implantation might shed some light on gene or protein expression changes if they are global and not restricted to cells of the conduction system. A recent publication has demonstrated the feasibility of focal gene transfer to modify electrical conduction in an animal model (21). If the molecular mechanisms were known, it might be possible to modify the expression of the genes that are dysregulated in human disease, either experimentally or, ultimately, for therapeutic purposes.

\section{Acknowledgments}

A. Maass is supported by a grant from the Deutsche Forschungsgemeinschaft (Ma 2185/1-1), and L.A. Leinwand is supported by NIH grant HL-50560.

1. Michalak, M., Corbett, E.F., Mesaeli, N., Nakamura, K., and Opas, M. 1999. Calreticulin: one protein, one gene, many functions. Biochem. J. 344:281-292.

2. Mesaeli, M., et al. 1999. Calreticulin is essentia for cardiac development. J. Cell Biol. 144:857-868.

3. Nakamura, K., et al. 2001. Complete heart block and sudden death in mice overexpressing calreticulin. J. Clin. Invest. 107:1245-1253.

4. Gutstein, D.E., et al. 2001. Conduction slowing and sudden arrhythmic death in mice with cardiac-restricted inactivation of Connexin 43. Circ. Res. 88:333-339.

5. Hagendorff, A., Schumacher, B., Kirchhoff, S., Luederitz, B., and Willecke, K. 1999. Conduction disturbances and increased atrial vulnerability in connexin 40 -deficient mice analyzed by transesophageal stimulation. Circulation. 99:1508-1515

6. Johnson, S., Michalak, M., Opas, M., and Eggleton, P. 2001. The ins and outs of calreticulin: from the ER lumen to the extracellular space. Trends Cell Biol. 11:122-129.

7. Rauch, F., Prud'homme, J., Arabian, A., Dedhar, S., and St-Arnaud, R. 2000. Heart, brain, and body wall defects in mice lacking calreticulin. Exp. Cell Res. 256:105-111.

8. Coppolino, M.G., et al. 1997. Calreticulin is essential for integrin-mediated calcium signaling and cell adhesion. Nature. 386:843-847.

9. Kuo, C.T., et al. 1997. GATA4 transcription factor is required for ventral morphogenesis and heart tube formation. Genes Dev. 11:1048-1060.

10. de la Pompa, J.L., et al. 1998. Role of the NF-ATc transcription factor in morphogenesis of cardiac valves and septum. Nature. 392:182-186.

11. Ranger, A.M., et al. 1998. The transcription factor NF-ATc is essential for cardiac valve formation. Nature. 392:186-190.

12. Sato, Y., et al. 1998. Cardiac-specific overexpression of mouse cardiac calsequestrin is associated with depressed cardiovascular function and hypertrophy in transgenic mice. J. Biol. Chem. 273:28470-28477.

13. Hunter, J.J., and Chien, K.R. 1999. Signaling pathways for cardiac hypertrophy and failure. N. Engl. J. Med. 341:1276-1283.

14. Houser, S.R., Piacentino, V., and Weisser, J. 2000. Abnormalities of calcium cycling in the hypertrophied and failing heart. J. Mol. Cell. Cardiol. 32:1595-1607.

15. Freeman, K., et al. 2001. Alterations in cardiac adrenergic signaling and calcium cycling differentially affect the progression of cardiomyopathy. J. Clin. Invest. 107:967-974.

16. Minamisawa, S., et al. 1999. Chronic phospholamban-sarcoplasmic reticulum calcium ATPase interaction is the critical calcium cycling defect in dilated cardiomyopathy. Cell. 99:313-322.

17. Molkentin, J., and Dorn, G., II. 2001. Cytoplasmic signaling pathways that regulate cardiac hypertrophy. Annu. Rev. Physiol. 63:391-426.

18. Wier, W.G., and Balke, C.W. 1999. $\mathrm{Ca}^{2+}$ release mechanisms, $\mathrm{Ca}^{2+}$ sparks and local control of excitation-contraction coupling in normal heart muscle. Circ. Res. 85:770-777.

19. Orth, T., Dorner, T., Meyer zum Bueschenfelde, K.H., and Mayet, W.J. 1996. Complete congenital heart block is associated with increased autoantibody titers against calreticulin. Eur.J. Clin. Invest. 26:205-215.

20. Moak, J.P., et al. 2001. Congenital heart block: development of late-onset cardiomyopathy, a previously underappreciated sequela. J. Am. Coll. Cardiol. 37:238-242.

21. Donahue, K.J., et al. 2000. Focal modification of electrical conduction in the heart by viral gene transfer. Nat. Med. 6:1395-1398. 